\title{
Investigation of the Adaptability of Transient Stability Assessment Methods to Real-
} Time Operation

Weckesser, Johannes Tilman Gabriel; Jóhannsson, Hjörtur; Sommer, Stefan; Østergaard, Jacob

Published in:

IEEE PES Innovative Smart Grid Technologies Conference Europe

Link to article, DOI:

10.1109/ISGTEurope.2012.6465835

Publication date:

2012

Document Version

Early version, also known as pre-print

Link back to DTU Orbit

Citation (APA):

Weckesser, J. T. G., Jóhannsson, H., Sommer, S., \& Østergaard, J. (2012). Investigation of the Adaptability of Transient Stability Assessment Methods to Real-Time Operation. In IEEE PES Innovative Smart Grid Technologies Conference Europe IEEE. https://doi.org/10.1109/ISGTEurope.2012.6465835

\section{General rights}

Copyright and moral rights for the publications made accessible in the public portal are retained by the authors and/or other copyright owners and it is a condition of accessing publications that users recognise and abide by the legal requirements associated with these rights.

- Users may download and print one copy of any publication from the public portal for the purpose of private study or research.

- You may not further distribute the material or use it for any profit-making activity or commercial gain

- You may freely distribute the URL identifying the publication in the public portal 


\title{
Investigation of the Adaptability of Transient Stability Assessment Methods to Real-Time Operation
}

\author{
Tilman Weckesser, Student Member, IEEE, Hjörtur Jóhannsson, Member, IEEE, \\ Stefan Sommer, Student Member, IEEE, Jacob Østergaard, Senior Member, IEEE
}

\begin{abstract}
In this paper, an investigation of the adaptability of available transient stability assessment methods to real-time operation and their real-time performance is carried out. Two approaches based on Lyapunov's method and the equal area criterion are analyzed. The results allow to determine the runtime of each method with respect to the number of inputs. Furthermore, it allows to identify, which method is preferable in case of changes in the power system such as the integration of distributed power resources (DER). A comparison of the performance of the analyzed methods leads to the suggestion that matrix reduction and time domain simulation are the most critical operations.
\end{abstract}

Index Terms-Power system stability, Stability analysis, Lyapunov method, Wide area measurements, Phasor measurement units

\section{INTRODUCTION}

$\mathbf{O}$ $\mathrm{NE}$ of the fundamental requirements of a modern society is a stable and secure operation of the electric power system and this is not expected to change in the future. In many countries a shift from fossil energy sources to renewable energy sources is taking place. In order to reach the future vision of a danish society with minimal dependency on fossil fuels [1], the power system faces a great challenge. It has to evolve into a system with minimal environmental impact, while continuing to provide its service satisfactory and at a competitive price. Therefore, a big share of the power production will use non-controllable sources, such as wind and solar radiation [1].

This will lead to increased fluctuations of the power system's operating point, which will make the planning of a stable and secure operation a challenging task, and a planning hours ahead may no longer be feasible. Hence, the state-of-the-art stability assessment methods, which are based on computationally demanding off-line calculations, will no longer be sufficient. A need for real-time assessment tools will arise.

Smart grid solutions are often suggested for coping with the fluctuating nature of wind and photo-voltaic power generation, where control of distributed energy resources is used for power balancing purpose. It is of paramount importance, that the smart grid control actions do not cause other stability problems in the system, while solving the power balancing problem. The

The authors are with the Centre for Electric Technology, Department of Electrical Engineering, Technical University of Denmark, 2800 Lyngby, Denmark. (email: jtgw@elektro.dtu.dk)

This research was supported by the Danish Strategic Research Council. need for real-time stability information in a future smart grid power system was accentuated e.g. in [2].

The phasor measurement technology [3]-[5] is seen as the enabling technology for the development of real-time wide area monitoring and control applications [6], [7].

Recent publications focus on the task of on-line security and stability assessment by utilizing synchronized phasor measurements. In [8], [9] an entirely new approach was developed for real-time assessment of the power system. However, in [10], [11] an existing method, used for off-line stability assessment, was adapted to real-time operation.

The potentials of adapting existing off-line assessment methods are further explored in this paper, where the focus is on methods for transient stability assessment. The computational complexity of several methods is investigated to identify the approaches that are well suited for real-time operation. This complexity investigation as well applies to future systems with changed topology. In contrast, an analysis based on simulations would only be representative for a current system.

\section{OVERVIEW OF EXISTING METHODS}

Figure 1 shows a mind-map of available transient stability assessment methods and depicts as well how research gradually developed towards more specific assessment methods. In the following, two approaches for transient stability assessment are discussed.

\section{A. Lyapunov's Direct Method}

Direct methods for transient stability assessment using Lyapunov functions have been developed since the 1960s (e.g. [12], [13]). The challenges are to determine a Lyapunov function, which represents the system with satisfactory accuracy, and, furthermore, to determine the most suitable critical energy, which allows the evaluation of the transient stability.

1) Transient Energy Function: A widely used energy function was derived in [14], which is nowadays most often referred to as transient energy function (TEF) (e.g. [16], [37]). This function is used in scalar Lyapunov approaches to assess transient stability in the power system. 


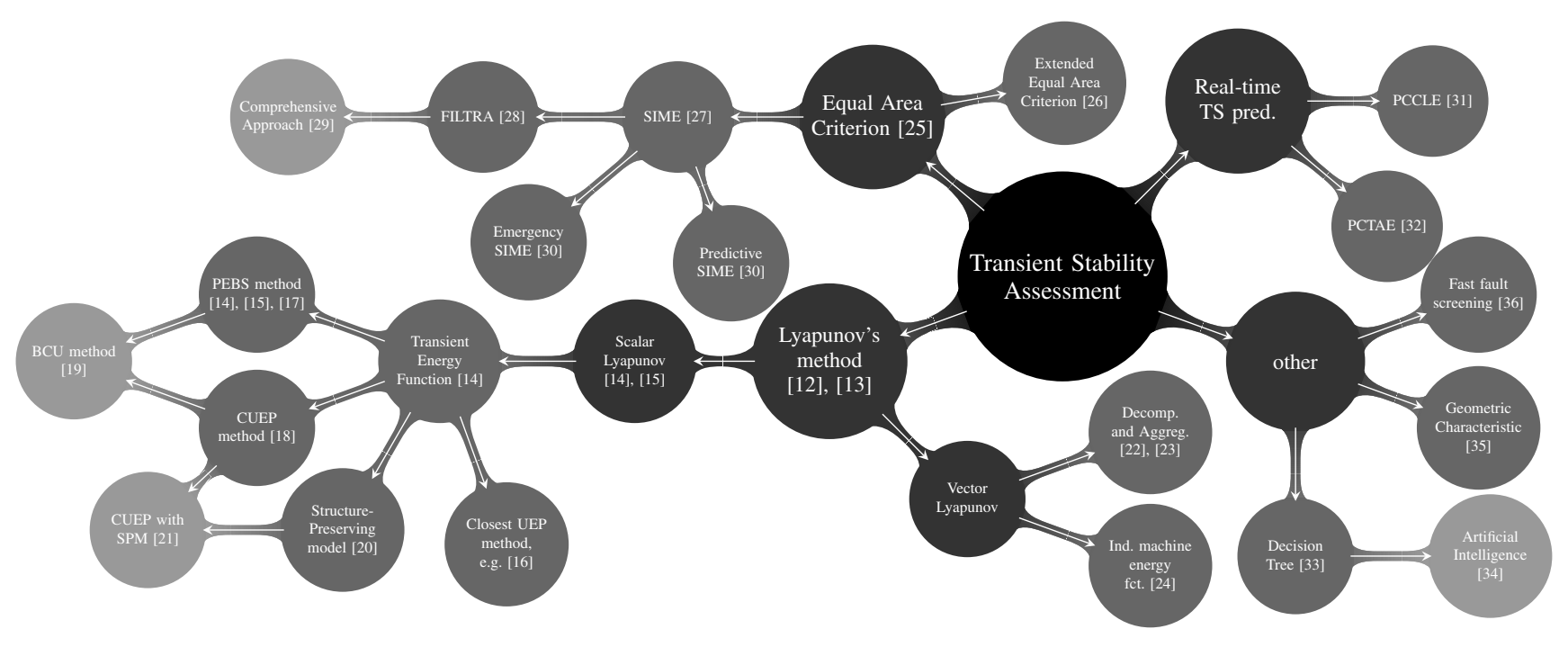

Fig. 1. Available methods and their development steps (not intended to be exhaustive)

$V=\sum_{i=1}^{n}(\overbrace{\left(\frac{1}{2} M_{i} \tilde{\omega}_{i}^{2}\right.}^{E_{\text {kin }}=}-\overbrace{P_{i}\left(\theta_{i}-\theta_{i}^{s}\right)}^{E_{\text {pot }, r}=})-\sum_{i=1}^{n-1} \sum_{j=i+1}^{n}[\underbrace{C_{i j}\left(\cos \theta_{i j}-\cos \theta_{i j}^{s}\right)}_{E_{m a g}}$

$-\underbrace{\int_{\theta_{i}^{s}+\theta_{j}^{s}}^{\theta_{i}+\theta_{j}} D_{i j} \cos \theta_{i j} d\left(\theta_{i}+\theta_{j}\right)}_{E_{d i s}}]$

$E \quad$ Constant voltage behind the transient reactance

$G_{i i} \quad$ Driving point conductance

$P_{m i} \quad$ Mechanical power input of the $i^{t h}$ machine

$V \quad$ Transient Energy

The terms in eq. (1) can be interpreted as:

- $E_{k i n}$ : Total change in rotor kinetic energy with respect to the center of inertia $(\mathrm{COI})\left(M_{i}\right.$ and $\tilde{\omega}_{i}$ are the inertia constant and angular velocity relative to COI respectively)

- $E_{p o t, r}$ : The change in potential energy of the rotor with respect to COI $\left(P_{i}=P_{m i}-E_{i}^{2} G_{i i}\right.$ and $\theta_{i}$ represents the rotor angle relative to the COI, the index $s$ denotes conditions at the stable equilibrium point (SEP))

- $E_{m a g}$ : Change in stored magnetic energy in the branch connecting machine $i$ and $j\left(C_{i j}\right.$ is the product of the voltages $E_{i}, E_{j}$ and the transfer susceptance $B_{i j}$ )

- $E_{d i s}$ : Change in dissipated energy of the branch connecting machine $i$ and $j$ ( $D_{i j}$ is the product of the respective voltages $E_{i}, E_{j}$ and the transfer conductance $G_{i j}$ )

The term $E_{d i s}$ causes that the total energy for a system with transfer conductances cannot be expressed in a closed form expression. One way to circumvent the problem is to neglect those when determining the reduced admittance matrix. The admittance matrix includes the transmission line impedances and loads, which are modeled as constant impedances. Neglecting the resistances is generally not acceptable [14].

2) Lyapunov Function: In order to use Lyapunov's method and the transient energy function, it has to be shown that eq. (1) exhibits the properties of a Lyapunov function.
Moreover, the power system has to have an asymptotic stability characteristic to be transient stable. In order to prove asymptotic stability with the Lyapunov method, the corresponding function $V(x)$ has to be a strong Lyapunov function $(V(x)>0 ; \dot{V}(x)<0)$ [38]. This can be relaxed to $\dot{V}(x) \leq 0$, if LaSalle's invariance principle is invoked and the stability of (1) $V(x)$ is only analyzed around the origin [37], [38].

The transient energy function (1) exhibits these characteristics, when using the classical model and assuming certain constraints such as neglecting the transfer conductances $G_{i j}=0$, [16], [39]. The Lyapunov function is then.

$$
V=\sum_{i=1}^{n}\left(E_{k i n}\left(\tilde{\omega}_{i}\right)-E_{\text {pot }, r}\left(\theta_{i}\right)\right)-\sum_{i=1}^{n-1} \sum_{j=i+1}^{n} E_{m a g}\left(\theta_{i j}\right)(2)
$$

3) Accounting for transfer conductances: Several approaches were developed to account for or to approximate the effects of the transfer conductances and only a few will be mentioned in the following. For a more complete discussion of the different approaches the reader is referred to [16].

a) Numerical integration: Some approaches utilize numerical integration to approximate the effects of transfer conductances. This additional term may cause the loss of the Lyapunov properties of the function. Furthermore, different integration paths may lead to different assessment results.

In [14] and [37] a linear approach is assumed and the additional term is integrated using the trapezoidal rule.

$$
\sum_{i=1}^{n-1} \sum_{j=i+1}^{n} \int_{\theta_{i}^{s}+\theta_{j}^{s}}^{\theta_{i}+\theta_{j}} D_{i j} \cos \theta_{i j} d\left(\theta_{i}+\theta_{j}\right) \equiv \sum_{i=1}^{n-1} \sum_{j=i+1}^{n} I_{i j}
$$

where

$$
I_{i j}=D_{i j} \frac{\theta_{i}+\theta_{j}-\theta_{i}^{s}-\theta_{j}^{s}}{\theta_{i j}-\theta i j^{s}}\left(\sin \theta_{i j}-\sin \theta_{i j}^{s}\right)
$$

Another approach was chosen by the authors of [15], here the sustained fault trajectory in the relative angle space determines the integration path [16]. 
b) Structure Preserving Model: A third approach was described by Bergen and Hill in [20], where the loads are preserved in the reduced admittance matrix, instead of being absorbed by it. Contrary to neglecting the transfer conductances of the transmission lines, neglecting the real part of the load impedances may not be a valid assumption [16].

In this approach the authors are considering a frequency dependent load (FDL) model, which load variations are linear about nominal frequency. This leads to a modification of the swing equation considered for the derivation of the TEF (eq. (1)) and, eventually, to a modified Lyapunov function [40].

The structure preserving model was further developed by other research groups (see also [16], [40]).

4) Critical Energy: In order to determine the stability of the system, the critical energy has to be computed. A straightforward theoretical approach to identify the critical energy is described in [16]: Step 1: Find all the unstable equilibrium points (UEPs) surrounding the new SEP of the post-fault system; Step 2: Define the critical energy $V_{\text {crit }}$ as the value corresponding to the UEP 'closest' to the SEP (lowest value). This theoretical approach is not feasible and several practical attempts were made to identify the critical energy.

a) Closest UEP approach: In [16] only all type-1 UEPs are considered to determine the closest unstable equilibrium and the corresponding critical energy. These are the operating points, where one of the machines looses synchronism. In order to identify the type-1 UEPs, an operating point of the following structure can be chosen as a starting point.

$$
\theta^{s j}=\left[\theta_{1, n}^{s}, \theta_{2, n}^{s}, \ldots, \pi-\theta_{j, n}^{s}, \ldots, \theta_{n-1, n}^{s}\right]
$$

The critical energy can be approximated by:

$$
V_{\text {crit }}=\min _{j=1, \ldots, n-1} V\left(\theta^{s j}, 0\right)
$$

This approach reduces the computational time, but does not have an effect on the conservativeness of the method.

b) Controlling Unstable Equilibrium Point (CUEP): The method of the controlling UEP was first considered by Fouad et al. [41]. The controlling UEP is the unstable equilibrium, which is closest to the particular trajectory of the disturbed system. At the UEP, in order to satisfy the swing equation (see e.g. [42]), the angular speed is equal to zero and, consequently, the critical energy in that point is determined by eq. (1) and (3) as follows.

$$
V_{\text {crit }}=-\sum_{i=1}^{n} E_{\text {pot }, r}\left(\theta_{i}^{u}\right)-\sum_{i=1}^{n-1} \sum_{j=i+1}^{n}\left[E_{\text {mag }}\left(\theta_{i j}^{u}\right)+I_{i j}^{u}\right]
$$

In order to identify the controlling UEP Fouad et al. propose in [18] a criterion as follows: "The post-disturbance trajectory approaches (if the disturbance is large enough) the controlling u.e.p. This is the u.e.p. with lowest normalized potential energy margin at the instant the disturbance is removed." This criterion can be computed using the following:

$$
\Delta V_{P E, \text { norm }}=\Delta V_{P E} / V_{K E, \text { corr }}
$$

where the energy absorbing capacity of the post-disturbance system $\Delta V_{P E}$ can be determined as the difference between potential energy at the UEP and at fault clearance. The equations for determining the corrected kinetic energy $V_{K E \text {,corr }}$ are provided in [18]. The correction is necessary due to the finding that not all the kinetic energy contributes to the separation of the critical generators [37], [41], [43].

In order to determine the minimum normalized potential energy margin $\Delta V_{P E, n o r m}$, the actual values of $\theta^{u}$ for the possible UEPs have to be known. In [18] it is proposed, that approximate values of $\theta^{u}$ are used, which can be obtained from the knowledge of the post-disturbance stable equilibrium point. After identification of the candidate CUEP the corresponding $\theta^{u}$ is calculated accurately and, eventually the critical energy corresponding to the CUEP can be calculated utilizing eq. (7). The critical energy computed can then be compared to the value of the energy function at the time of fault clearance and the stability of the system can be determined.

A more detailed description of the approach is provided in [18] or [39]. This approach clearly reduces the conservativeness of the method compared to the closest UEP method.

c) Potential Energy Boundary Surface (PEBS): Kakimoto et al. [15] as well as Athay et al. [14] proposed to determine the transient stability region by analyzing the potential energy function $\left(V_{p}\right)$. According to [14] the $V_{p}$ has within a periodic frame of rotor angles at most one relative minimum (new SEP). The rest of the extreme values correspond to relative maxima and saddle-points (UEPs). The authors of [15] propose a stability boundary, where the saddle-points surrounding the SEP are connected through curves $\left(O_{x}\right)$, which are orthogonal to equipotential curves $\left(V_{p}(\theta, E)=C_{i}\right)$. The authors argue that, due to the fact that the direction of the total torque applied to the system is orthogonal to the equipotential curves, the system will loose synchronism after crossing a curve $O_{x}$. In [15] it is suggested that the fault-on trajectory is simulated until it crosses a curve $O_{x}$. The point of crossing the boundary can be detected by analyzing the time derivative of the kinetic energy.

A theoretical foundation of the PEBS method was later presented by Chiang et al. in [17]. The authors define the PEBS as the stability boundary of the gradient system

$$
\dot{\theta}=-\partial V_{p} / \partial \theta
$$

and conclude that it is a local approximation of the stability boundary of a power system described by the swing equation.

d) Boundary of stability region based controlling unstable equilibrium point method (BCU method): The BCU method, described in [19], is based on the relationship of the stability boundary of the original and the reduced system determined by eq. (9) [17]. Where the reduced system is described by:

$$
\dot{\theta}_{i n}=\left(P_{i}-P_{e i}\right)-\left(M_{i} / M_{n}\right)\left(P_{n}-P_{e n}\right)
$$

$P_{e i} \quad$ Injected electrical power of the $i$ th machine

Roughly speaking, the BCU method analyzes the fault-on trajectory and detects when the boundary of the reducedstate model is crossed. This point is called exit point and is 
used as the initial condition when integrating the post-fault reduced system. The integration yields a new approximation of $\theta$ corresponding to the controlling UEP and is used as an initial guess to find the actual CUEP by solving eq. (9) equal to zero. The transient stability of the system can then be determined utilizing the CUEP, provided by the BCU method, and applying the CUEP method described in section II-A4b.

\section{B. Methods based on the Equal Area Criterion (EAC)}

The equal area criterion was developed in the late 1930's, though the origin is not really clear [30].

1) Equal Area Criterion (EAC): The method allows the determination of transient stability of an one-machine infinite bus system (OMIB) without computing the swing curves. Therefore, the following simplifications and assumptions were considered. The synchronous machine (SM) is represented by a constant voltage magnitude behind the synchronous reactance and the mechanical power input is assumed to be constant. Furthermore, the damping of the machine is neglected and the loads are represented by constant impedances. It was later shown that the simplifications can be relaxed [30].

When the damping is neglected and after manipulation, the swing equation can be written for an OMIB as follows [44]:

$$
[d \delta / d t]^{2}=\int(2 / M)\left(P_{m}-P_{e}\right) d \delta
$$

$P_{e / m}$ Electric power injection / mechanical power input

$\delta \quad$ Relative rotor angle

Initially, the derivative of the angle is zero and changes after the disturbance. For a stable case the angle reaches a maximum at $\delta_{m}$ and changes the direction thereafter. From this the following stability criteria can be formulated [44].

$$
\int_{\delta_{0}}^{\delta_{m}}(2 / M)\left(P_{m}-P_{e}\right) d \delta=0
$$

The integral can be split up into two areas, which can be calculated as follows (indices $D$ and $P$ denote conditions during and after fault clearance respectively).

$$
\begin{aligned}
& A_{a c c}=\int_{\delta_{0}}^{\delta_{c}}\left(P_{m D}-P_{e D}\right) d \delta=\int_{\delta_{0}}^{\delta_{c}} P_{a} d \delta \\
& A_{d e c}=-\int_{\delta_{c}}^{\delta_{m}}\left(P_{m P}-P_{e P}\right) d \delta=-\int_{\delta_{c}}^{\delta_{m}} P_{a} d \delta
\end{aligned}
$$

$P_{a} \quad$ Accelerating power

A stability margin $\eta$ can then be determined as follows, where $\delta_{u}$ is the rotor angle at the UEP.

$$
\eta=-\int_{\delta_{0}}^{\delta_{u}} P_{a} d \delta=A_{d e c}\left(\delta_{m}=\delta_{u}\right)-A_{a c c}
$$

A positive margin represents a stable case, a negative an unstable and in the case that $\eta=0$ it is a critical case.

2) Extended Equal Area Criterion (EEAC): In order to determine the stability of the power system as a response to a certain disturbance, the method described in [26] decomposes the multi-machine system into a set of critical machine(s) and a set of the 'remaining' generators. The machines in the two groups are aggregated and then transformed into two equivalent machines to form an OMIB system. Furthermore, in order to achieve simple algebraic equations for the assessment criteria, a modified Taylor series expansion is applied to determine the rotor angle. a) Aggregation of Machines and Formulation of the OMIB system: The two sets of machines are aggregated as follows.

$$
M_{a, s}=\sum_{i \in A, S} M_{i} ; \delta_{a, s}=M_{a, s}^{-1} \sum_{i \in A, S} M_{i} \delta_{i} ;
$$

where $S$ is the set of the machines of the critical cluster; $s$ is its equivalent aggregated to one machine and $t_{0}^{+}=0^{+}$is the time immediately following the disturbance. In the same way the 'remaining' machines can be aggregated, where $A$ is the set of the 'remaining' machines; $a$ is its equivalent aggregated to one machine. The OMIB system is then described by

$$
M \ddot{\delta}=P_{m}-P_{e}=P_{m}-\left[P_{c}+P_{\max } \sin (\delta-v)\right]
$$

The equations for $P_{m}, P_{c}, P_{\max }$ and $v$ can be found in [26].

b) Determine acceleration and deceleration area: The two areas can be computed as follows.

$$
\begin{aligned}
& A_{a c c}=\left(P_{m}-P_{c D}\right)\left(\delta_{\tau}-\delta_{0}\right) \\
& +P_{\max D}\left[\cos \left(\delta_{\tau}-v_{D}\right)-\cos \left(\delta_{0}-v_{D}\right)\right] \\
& A_{d e c}=\left(P_{c P}-P_{m}\right)\left(\pi-\delta_{\tau}-\delta_{P}+2 v_{P}\right) \\
& +P_{\max P}\left[\cos \left(\delta_{\tau}-v_{P}\right)-\cos \left(\delta_{P}-v_{P}\right)\right]
\end{aligned}
$$

c) Critical Clearing Time (CCT): In order to determine the CCT, the rotor angle is expressed in a Taylor series.

$$
\delta_{\tau}=\delta_{0}+\alpha_{1}^{-1} \alpha_{2}^{-2} \gamma\left(\tau^{2} / 2\right)+\alpha_{1}^{-1} \alpha_{2}^{-4} \ddot{\gamma}\left(\tau^{4} / 24\right) ; \gamma=\ddot{\delta}
$$

where $\alpha_{1,2}$ are corrective factors to compensate for the truncation error [26]. The Taylor series expansion solely contains even derivatives of $\delta$, this is due to continuity which dictates that $\dot{\delta}$ is equal to zero at $t_{0}^{+}$and that causes the cancellation of all higher-order odd derivatives of $\delta$ [26].

The stability of the power system with respect to a certain contingency can be assessed by determining the CCT. Therefore, (18) and (19) are solved for $\delta_{c}$ and (20) is utilized to calculate $t_{c}$. A simple comparison of the CCT and the actual clearing time yields the stability assessment result.

d) Identification Critical Machine(s): In order to identify a (cluster of) critical machine(s), the authors of [26] suggest the acceleration criterion $\sigma_{i}$.

$$
\sigma_{i}=\left(P_{m i}-P_{e i}\left(\delta\left(t_{0}^{+}\right)\right)\right) / M_{i}
$$

The machines with the largest acceleration criterion are the critical machines. Critical groups can be identified, when considering the CCTs of the machines and their interconnection. A critical group is formed if two to three generators at the top of the list have similar CCTs and are strongly interconnected.

3) SIME: SIngle Machine Equivalent (SIME) poses an hybrid approach, which combines the advantages of a direct method and the time domain simulation approach.

In [30] different applications of the method are described. For fast stability assessment the predictive SIME seems to be appropriate and is considered in this analysis.

In the following a short description of the method is given. It solely comprises the most important features and the reader is referred to [27], [30] for a complete description. 
a) Identification of Critical Machines: In order to identify the critical machines, the rotor angles of the generators are analyzed in every time step of the post-fault time domain simulation. In [28], [30] the generators are sorted according to their rotor angles and the angles of consecutive generators are compared to detect the 'maximum gap'. The generators above each gap are considered to form the set of critical generators and a corresponding candidate OMIB is formed. The timedomain simulation is continued until a candidate OMIB fulfills the instability criteria stated in (22).

b) Formulation of OMIB: After the critical machines are identified the system generators can be transformed into two equivalent machines and the OMIB system can be set up. The inertia coefficients of the equivalent machines can be calculated utilizing (16). The OMIB can then be described by (17), after $P_{m}, P_{c}, P_{\max }$ and $v$ are determined.

c) Stability criterion: In [27] the stability criteria for the OMIB trajectories are determined as follows.

Unstable trajectory is a trajectory, which reaches an angel $\delta_{u}$ at time $t_{u}$ where

$$
P_{a}\left(t_{u}\right)=0 ; d P_{a} /\left.d t\right|_{t=t_{u}}>0
$$

Stable trajectory is a trajectory, which reaches an angel $\delta_{r}<\delta_{u}$ at time $t_{r}$, where $\omega\left(t_{r}\right)=0 ; P_{a}\left(t_{r}\right)<0$.

d) Stability margin: The stability margin can then be determined as follows.

$$
\eta=-\int_{\delta_{i}}^{\delta_{u}} P_{a} d \delta-(1 / 2) M \omega_{i}^{2}
$$

$\delta_{i}=\delta\left(t_{i}\right)$ OMIB angle at time $t_{i}$ short after fault clearance

In order to determine the stability margin, a weighted leastsquare approximation (WLS) is used in [30] and $P_{a}(\delta)$ is extrapolated between $\delta_{r}$ to $\delta_{u}$. The parameters $a, b$ and $c$ in eq. (24) are determined using values of $P_{a}$ at three successive time steps

$$
P_{a}(\delta)=a \delta^{2}+b \delta+c
$$

e) Predictive SIME: In [30] the predictive SIME method is used within a closed emergency control loop. The method is assumed to receive real-time measurements, which contain the rotor angles, speeds and accelerations. Subsequently, the method is used to carry out a predictive stability assessment, which can be used to determine appropriate control actions.

The procedure of the predictive SIME is described in [30] as follows. Step 1: At a time shortly after the disturbance clearance, consider three successive incoming measurements of the individual machines. Step 2: Use Taylor series to predict machine angles some time ahead and identify critical machines as described in section II-B3a. Step 3: Construct the corresponding OMIB as described in section II-B3b. Step 4: Determine OMIB parameters from the received measurements. Step 5: Approximate $P_{a}-\delta$ curve with WLS approximation. Step 6: Solve eq. (24) to determine $\delta_{u}$. Step 7: Determine stability margin as described in section II-B3d.

f) Modification of Predictive SIME: In order to use the predictive SIME method for a fast stability assessment, the real-time measurements are used as initial conditions for a time domain simulation. The simulation is run until shortly

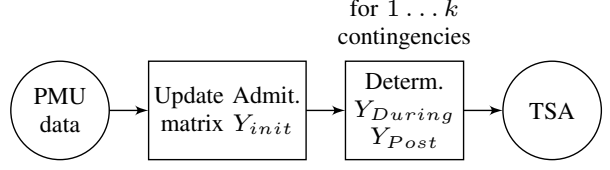

Fig. 2. PMU data utilization

TABLE I

COMPLEXITY ESTIMATION, $b$ : NUMBER OF BRANCHES; $m$ :NUMBER OF GENERATORS; $n$ : NUMBER OF BUSES; $k$ : NUMBER OF CONTINGENCIES

\begin{tabular}{|l|r|r|r|}
\hline Function & Time & Frequency & \\
\hline \hline PMU data & $O(n+b)$ & 1 & $O(n+b)$ \\
\hline Admittance matrix & $O(m+n+b)$ & 1 & $O(m+n+b)$ \\
\hline$Y_{\text {During }}, Y_{\text {Post }}$ & $O(m+n+b)$ & $k$ & $k O(m+n+b)$ \\
\hline \hline TOTAL & & & $k O(m+n+b)$ \\
\hline
\end{tabular}

after the disturbance is cleared and the simulated values are used instead of the measured values in the predictive SIME.

The stability analysis can then be carried out following the steps described in the previous section.

\section{RESUltS}

The runtime analysis of the examined methods are presented in the following. It is assumed that PMU data providing full observability of the system are received in real-time.

The complexity of the algorithms of the distinct methods are compared in the following. This estimation should be valid for future power system and, consequently, the effects of structural changes, such as the increasing installation of DER, should be reflected in the results. Therefore, the algorithms of the different methods are compared using the big $O$-notation, which is common practice when analyzing algorithms [45]. If simulations were carried out instead, the analysis would be tied to the particular test case, and effects of structural changes would not be accounted for. A simulation of a relatively small test power system such as the IEEE test system cases would assess runtime on current power systems, and the results would fail to capture dependence on the system size and topology.

\section{A. PMU data utilization}

The procedure to extract the needed information from the received PMU measurements is shown in figure 2. It is assumed that voltage measurements from each node are received as well as current measurements from both ends of each branch. These measurements are used to update the admittance matrix and with a list of contingencies the admittance matrices for the during- and post-fault condition are set up.

The matrices are extended by the internal nodes of the machines behind their transient reactance $X_{d}^{\prime}$, since some of the methods assume the voltages behind $X_{d}^{\prime}$ to be constant.

The number of operations to update these matrices is proportional to the number of branches, nodes and machines in the system. The results in $O$-notation are shown in table I.

\section{B. Scalar Lyapunov's Direct method}

The discussed methods assessing transient stability using Lyapunov's method are only differing in the way the critical 


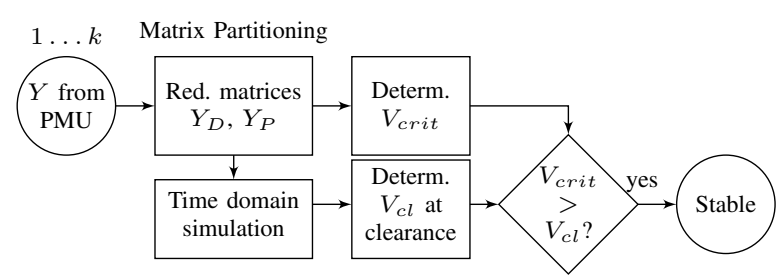

Fig. 3. Flow diagram scalar Lyapunov method

transient energy is determined. Therefore, the general procedure is shown in fig. 3 and in subsequent sections it is solely described how each method calculates the critical energy.

The methods based on a scalar Lyapunov function use the reduced admittance matrix, which means that the nodes where no current enters or leaves the network are eliminated. This elimination is carried out using matrix algebra and partitioning of the admittance matrix [46]. The most complex step, in determining the reduced admittance matrix, is to find the inverse of the $(n \times n)$ admittance matrix. However, the matrix is highly sparse, which enables to determine the inverse much faster than for the case of a full matrix. In order to use the algorithm proposed by Takahishi et al. [47], the matrix has to be transformed into a banded matrix by applying the reverse Cuthill-McKee algorithm, which complexity was shown in [48] to be proportional to the number of edges and, hence, proportional to $O(b)$. The complexity of calculating the whole inverse of a banded matrix can be approximated by $O\left(n^{2} d\right)$ [49], where $d$ is the bandwidth. It can be argued that in the case of power system networks the bandwidth of the ordered admittance matrix is small compared to the number of nodes and in large system smaller than the number of machines. In the IEEE 14-bus system $d$ is larger than $m$. In the IEEE 300bus system the bandwidth is considerably smaller than the number of machines (d: $35, \mathrm{~m}: 68$ ).

In order to determine the transient energy of the system at the point of fault clearance, a time domain simulation of the whole system until the clearance has to be carried out. An explicit integration method such as Runge-Kutta (R-K) method is used to calculate the time response. In order to carry out the time domain simulation a set of algebraic equations and differential equations has to be solved. When utilizing the R-K method the complexity of solving the differential equation is proportional to $O\left(\mathrm{~m}^{2}\right)$. In order to solve the algebraic equation fast the sparsity of the admittance matrix is utilized and corresponding fast algorithms are used. By performing a LU-factorization and through forward and backward substitution the process can be considerably accelerated. The LU-factorization is, however, the operation with the highest computational cost and can be approximated by $O\left(d^{2} n\right)$ [49].

After the values of the state variables at fault clearance are determined, the transient energy of the system at that point in time can be determined. The complexity of calculating the transient energy itself is $O\left(\mathrm{~m}^{2}\right)$, due to the double sum (see eq. (1)). Additionally to the state variables at fault clearance, the state variables at the during-fault stable equilibrium need to be known. To find the SEP the Newton Raphson (N-R) method is used and this leads to a complexity of $O\left(\mathrm{~m}^{3}\right)$.
TABLE II

COMPLEXITY ESTIMATION PROCESSES SHARED BY THE TWO SCALAR LYAPUNOV METHODS, $d$ : BANDWIDTH OF BANDED MATRIX

\begin{tabular}{|l|r|r|r|}
\hline Function & Time & Freq. & \\
\hline \hline$Y$ from PMU & $O(m+n+b)$ & 1 & $O(m+n+b)$ \\
\hline Reduce Matrices & $O\left(d n^{2}\right)$ & 1 & $O\left(d n^{2}\right)$ \\
\hline Time domain simulation ${ }^{\dagger}$ & $O\left(d^{2} n\right)$ & $x$ & $x O\left(d^{2} n\right)$ \\
\hline Determine $V_{c l}$ & $O\left(m^{3}\right)$ & 1 & $O\left(m^{3}\right)$ \\
\hline Determine $V_{c r i t}$ & & & III-B1 \& III-B2 \\
\hline Compare $V_{c l}$ and $V_{c r i t}$ & 1 & 1 & 1 \\
\hline \hline TOTAL $^{\ddagger}($ one disturbance) & & & $O\left(d n^{2}\right)$ \\
\hline
\end{tabular}

$\diamond$ : Matrix reduction by partitioning of the matrix, see [46, section 2.1]

$\dagger$ : Using an explicit integration method Runge-Kutta with $x$ time steps

$\ddagger$ : Runtime evaluation without determination of the critical energy

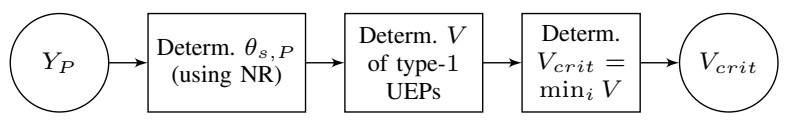

Fig. 4. Flow diagram determine $V_{\text {crit }}$ in the closest UEP method

TABLE III

COMPLEXITY ESTIMATION CLOSEST UEP

\begin{tabular}{|l|r|r|r|}
\hline Function & Time & Freq. & \\
\hline \hline Lyapunov method frame & $O\left(d n^{2}\right)$ & 1 & $O\left(d n^{2}\right)$ \\
\hline Determine $V$ of type-1 UEPs & $O\left(m^{3}\right)$ & $m$ & $O\left(m^{4}\right)$ \\
\hline Determine $V_{\text {crit }}$ (merge sort) & $O(m \log m)$ & 1 & $O(m \log m)$ \\
\hline \hline TOTAL(one disturbance) & & & $O\left(m^{4}\right)$ \\
\hline
\end{tabular}

$\diamond$ : see table II



Fig. 5. Flow diagram determine $V_{\text {crit }}$ in the BCU method

Finally, the determined transient energy at fault clearance and the critical energy of the post-fault system are compared.

1) Closest UEP: Figure 4 shows the block diagram of the closest UEP method, which determines the critical energy of the post-fault system by considering all type-1 UEP. The procedure to determine all $m$ type- 1 UEPs is the computational most expensive step, because the UEPs are identified using N$\mathrm{R}$ method which comprises the inversion of an $2 m \times 2 m$-matrix resulting in a complexity proportional to $O\left(\mathrm{~m}^{3}\right)$.

The next step to identify the lowest critical energy is fast with $O(m \log m)$, when applying the merge sort algorithm.

The runtime evaluation results are presented in tab. III.

2) BCU method: The procedure to determine the critical energy when applying the BCU method is depicted as block diagram in fig. 5. The corresponding estimation of the runtime is depicted in table IV. When applying an explicit integration method to carry out the time domain simulation, its complexity is proportional to the square of the number of machines in the system. The second time domain simulation, which is of the reduced system, comprises a reduced number of differential equation, the number of equations and the complexity to solve those is, however, still proportional to the number of machines squared $O\left(\mathrm{~m}^{2}\right)$. In both cases in each simulation step a set of algebraic equations has to be solved using LU-factorization with complexity proportional to $O\left(d^{2} n\right)$. 
TABLE IV

COMPLEXITY ESTIMATION BCU

\begin{tabular}{|c|c|c|c|}
\hline Function & Time & Freq. & \\
\hline Lyapunov method frame ${ }^{\diamond}$ & $O\left(d n^{2}\right)$ & 1 & $O\left(d n^{2}\right)$ \\
\hline Sim. until PEBS crossing & $O\left(d^{2} n\right)$ & $y$ & $y O\left(d^{2} n\right)$ \\
\hline Sim. reduced system ${ }^{\ddagger}$ & $O\left(d^{2} n\right)$ & $z$ & $z O\left(d^{2} n\right)$ \\
\hline Find CUEP & $O\left(m^{3}\right)$ & 1 & $O\left(m^{3}\right)$ \\
\hline Determine $V_{\text {crit }}$ & $O\left(m^{2}\right)$ & 1 & $O\left(m^{2}\right)$ \\
\hline TOTAL(one disturbance) & & & $O\left(d n^{2}\right)$ \\
\hline
\end{tabular}

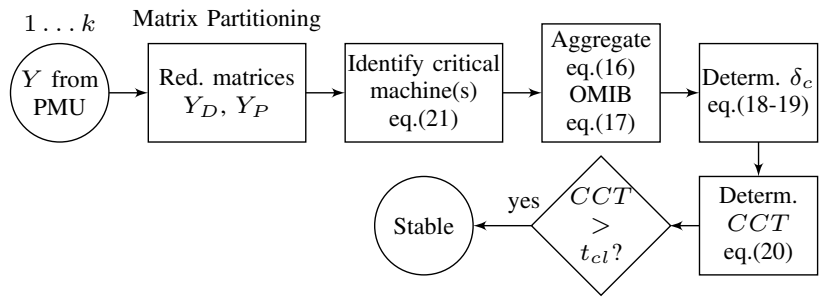

Fig. 6. Flow diagram extended equal area criterion

In order to find the controlling UEP, the N-R method is applied, where the results of the time domain simulation serve as an initial guess. The complexity of this step is consequently proportional to $O\left(\mathrm{~m}^{3}\right)$ and the calculation of $V_{\text {crit }}$ is proportional to $O\left(\mathrm{~m}^{2}\right)$.

\section{Equal Area Criterion Methods}

The equal area in its pure form is promising to be very fast due to the lack of time domain simulation, the results, however, are expected to be conservative, because of the assumed constant voltage magnitude behind the transient reactance frozen at the time just before the fault occurrence. The SIME method tries to overcome these challenges through the combination of a direct method with time domain simulation.

1) Extended Equal Area Criterion: Figure 6 shows the procedure to determine stability using the EEAC method depicted as a block diagram. The method utilizes the admittance matrices provided from the PMU data procedure. The matrices are reduced as described in the preceding section $\left(O\left(d n^{2}\right)\right)$. Following, the acceleration criterion is calculated for each machine, which allows to determine the critical machine(s). Therefore, the electrical power injection of each machine at fault occurrence has to be calculated $O\left(\mathrm{~m}^{2}\right)$.

The critical machines can then be identified after sorting the calculated acceleration criteria and identifying the machines with the largest values, this can be achieved by employing the merge sort algorithm $(O(m \log m))$.

In the next step the machines are aggregated into two equivalent machines and the OMIB system is determined. The number of operations associated with this process is proportional to the square of the number of machines.

Eventually, the critical angle and the CCT can be calculated and the results can be compared to the settings of the associated protection relays to evaluate stability. The results of the runtime assessment are shown in table $\mathrm{V}$.
TABLE V

COMPLEXITY ESTIMATION EEAC

\begin{tabular}{|l|r|r|r|}
\hline Function & Time & Freq. & \\
\hline \hline$Y$ from PMU & $O(m+n+b)$ & 1 & $O(m+n+b)$ \\
\hline Reduce Matrices & $O\left(d n^{2}\right)$ & 1 & $O\left(d n^{2}\right)$ \\
\hline Acceleration criteria & $O(m)$ & $m$ & $O\left(m^{2}\right)$ \\
\hline Identify critical & $O(m \log m)$ & 1 & $O(m \log m)$ \\
\hline Aggregate $\&$ form OMIB & $O\left(m^{2}\right)$ & 1 & $O\left(m^{2}\right)$ \\
\hline Determine $\delta_{c}, C C T$ & 1 & 1 & 1 \\
\hline Compare $t_{c l}$ and $C C T$ & 1 & 1 & 1 \\
\hline \hline TOTAL(one disturbance) & & & $O\left(d n^{2}\right)$ \\
\hline
\end{tabular}

$\diamond:$ Matrix reduction by partitioning of the matrix, see [46, section 2.1]

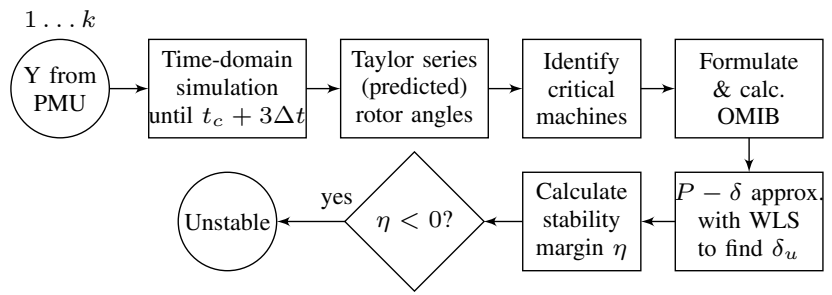

Fig. 7. Flow diagram modified predictive SIME

TABLE VI

COMPLEXITY ESTIMATION SIME

\begin{tabular}{|c|c|c|c|}
\hline Function & Time & Freq. & \\
\hline PMU data & $O(m+n+b)$ & 1 & $O(m+n+b)$ \\
\hline Time domain sim. $^{\dagger}$ & $O\left(d^{2} n\right)$ & $x$ & $x O\left(d^{2} n\right)$ \\
\hline Rotor angle (pred.) & 1 & $m$ & $O(m)$ \\
\hline Ident. critical mach. & $O(m \log m)$ & 1 & $O(m \log m)$ \\
\hline Aggregate \& form OMIB $\ddagger$ & $O(m)$ & 1 & $O(m)$ \\
\hline$P-\delta$ approx. & 1 & 1 & 1 \\
\hline Det. stability margin & 1 & 1 & 1 \\
\hline "TOTAL(one dist.) & & & $\overline{x O\left(d^{2} n\right)}$ \\
\hline
\end{tabular}

$\dagger$ : Explicit integration method (R-K method) with $x$ time steps

$\ddagger$ : Differs to EEAC see text for explanation

2) Modified Predictive SIME: Figure 7 shows the transient stability assessment using the SIME method in a block diagram. The first step in the method based on SIME is to carry out a time-domain simulation until three time steps after the fault clearance. For the time domain simulation a number of differential equations proportional to the number of machines in the system have to be solved using an explicit integration method. The complexity of the individual differential equations varies and in some cases, e.g. the derivative of the rotor speed depends on the remaining generators, which leads to a total complexity estimation proportional to $O\left(\mathrm{~m}^{2}\right)$. The second step in the time domain simulation is to update the network parameters, therefore $n$ algebraic equations have to be solved. Due to the fact that the admittance matrix is highly sparse special algorithms can be used to solve these equations and this results in a complexity approximated by $O\left(d^{2} n\right)$. In the following, the rotor angle of each individual machine advancing in time are predicted through a Taylor series expansion, where complexity is estimated to be proportional to $O(m)$. The predicted rotor angles are sorted and the critical machine candidates are identified through the biggest 'gap' (merge sort $O(m \log m)$ ). After identification of the critical machines, the machines can be aggregated and transformed into an OMIB system, this processes is less complex than in 
the EEAC method due to the availability of parameters from the time domain simulation. The $P-\delta$-curve is then approximated with the simulated data and equation (24). Thereupon, the angle $\delta_{u}$ and time $t_{u}$, which meet the conditions stated in eq. (22), are identified and used to determine the stability margin utilizing (23). Eventually, by evaluating the stability margin the stability can be determined. The overall complexity of the SIME method can then be approximated by $x O\left(d^{2} n\right)$.

\section{DISCUSSION}

The results in tab. I to VI make it possible to identify the dominant operation in all methods. The complexity of the processes of handling the PMU data and updating of the matrices is linear and negligible in all the analyzed methods.

In the analysis of the closest UEP method it was shown that identification of all the type-1 UEPs is the most complex step with an associated complexity proportionally $O\left(\mathrm{~m}^{4}\right)$. In the case of the BCU method and the EEAC the operation with the highest associated computational costs is the reduction of the admittance matrix $O\left(d n^{2}\right)$, which is for the case of the EEAC in good agreement with the results in [26]. The SIME method overcomes some of the challenges of the EEAC method by including time domain simulation. This results in a complexity proportional to $O\left(d^{2} n\right)$.

In direct comparison with the closest UEP method, the BCU method is faster and less conservative because of the determination of the CUEP. The use of Lyapunov's methods compared to the EEAC allows more detailed models of the system and the machines. While the EEAC is restricted to the classic model, more complex structure-preserving models can be utilized in Lyapunov's method. The SIME circumvents the restriction to the classical model by using a detailed time domain simulation. It should be noted that the needed simulation is shorter compared to the BCU method.

In the complexity analysis the time domain simulation was assumed to be carried out using an explicit integration method, this method is not A-stable and, consequently, requires a small integration time step [44]. An improvement of the accuracy of the time domain simulation and the usage of larger time steps could be enabled by employing an implicit integration method, which uses for example the trapezoidal rule. However, this may lead to an increase of the complexity to $x O\left(d n^{2}\right)$. Because the aim of the paper is to investigate the method's real-time capability, it was decided to use a faster but less accurate integration method.

The results of the analysis of the time domain simulation are as well dependent on the accuracy of the representation of the loads. For this analysis it was assumed that the impedances of the loads vary during the disturbance and, consequently, the admittance matrix needs to be updated and factorized in each simulation step. When load impedances are assumed to be constant, the simulation is still dominated by the LUfactorization, but it has to be carried out only two times.

Another critical issue is the comparison of complexities proportional to the number of machines and proportional to the number of nodes in the system. Nowadays, due to the centralized power generation, in a power system the number of machines is considerably lower than the number of nodes.
This ratio is to change in the future, due to increasing installation of DER. Consequently, the number of machines in the system could approach values closer to the number of nodes, which may lead to changes in the complexity evaluation, e.g. domination of the Lyapunov method frame by $O\left(\mathrm{~m}^{3}\right)$.

Under the consideration that in the future power system the number of machines in the system will be considerably higher than today, the analysis of the complexity suggest that the SIME method may provide the fastest stability evaluation of the system. However, an additional uncertainty is introduced due to the prediction of the rotor angles using a Taylor series and a weighted least-square approximation of the $P-\delta$-curve.

\section{CONCLUSION}

The paper begins with a review of methods using Lyapunov's method and the EAC, which are further analyzed.

When using the Lyapunov approach the challenge is to derive an appropriate energy function, which fulfills the criteria for a Lyapunov function, and to identify the appropriate critical energy for the stability evaluation. The BCU method combined with a structure-preserving model seems to overcome these challenges in a promising way.

The second approach uses the equal area criterion. The EEAC method allows to carry out the transient stability assessment without the need for time domain simulations, but requires the reduced admittance matrix and is limited to the use of the classical model, which leads to conservative results.

SIME overcomes some of the limitation of the EEAC method through the use of a detailed time domain simulation.

The results suggest that the methods utilizing the equal area criterion are faster than the methods using energy functions when assessing transient stability in large systems with distributed power generation. The SIME method may be the preferable tool to fast evaluate the transient stability of a large power system, due to the less conservative results.

\section{REFERENCES}

[1] The Danish Ministry of Climate and Energy, "Energy Strategy 2050 from coal, oil and gas to green energy," The Danish Ministry of Climate and Energy, Stormgade 2-6 1470 Copenhagen K Denmark, Tech. Rep., Feb. 2011.

[2] F. Li et al., "Smart Transmission Grid: Vision and Framework," IEEE Trans. Smart Grid, vol. 1, no. 2, pp. 168-177, Sep. 2010.

[3] A. Phadke, J. Thorp, and M. Adamiak, "A New Measurement Technique for Tracking Voltage Phasors, Local System Frequency, and Rate of Change of Frequency," IEEE Trans. Power App. Syst., vol. 102, no. 5, pp. 1025-1038, May 1983.

[4] A. Phadke and J. Thorp, Synchronized Phasor Measurements and Their Applications. Springer Verlag, 2008.

[5] A. Phadke and R. De Moraes, "The Wide World of Wide-Area Measurement," IEEE Power Energy Mag., vol. 6, no. 5, pp. 52-65, Sep. 2008.

[6] S. Skok, I. Ivankovic, and Z. Cerina, "Applications Based on PMU Technology for Improved Power System Utilization," in Power Engineering Society General Meeting, 2007. IEEE, Jun. 2007, pp. 1-8.

[7] D. Novosel et al., "Dawn of the grid synchronization," IEEE Power Energy Mag., vol. 6, no. 1, pp. 49-60, Jan. 2008.

[8] H. Jóhannsson, "Development of early warning methods for electric power systems," Ph.D. dissertation, 2011.

[9] H. Jóhannsson et al., "Real-time stability assessment based on synchrophasors," in Proceedings of 2011 IEEE Trondheim PowerTech, Jun. 2011, pp. 1-8.

[10] M. Glavic and T. Van Cutsem, "Wide-Area Detection of Voltage Instability From Synchronized Phasor Measurements. Part I: Principle," IEEE Trans. Power Syst., vol. 24, no. 3, pp. 1408-1416, Aug. 2009. 
[11] — "Wide-Area Detection of Voltage Instability from Synchronized Phasor Measurements. Part II: Simulation Results," IEEE Trans. Power Syst., vol. 24, no. 3, pp. 1417-1425, Aug. 2009.

[12] G. E. Gless, "Direct Method of Liapunov Applied to Transient Power System Stability," IEEE Trans. Power App. Syst., vol. PAS-85, no. 2, pp. 159-168, Feb. 1966.

[13] A. H. El-Abiad and K. Nagappan, "Transient Stability Regions of Multimachine Power Systems," IEEE Trans. Power App. Syst., vol. PAS85, no. 2, pp. 169-179, Feb. 1966.

[14] T. Athay, R. Podmore, and S. Virmani, "A practical Method for the Direct Analysis of Transient Stability," IEEE Trans. Power App. Syst., vol. PAS-98, no. 2, pp. 573-584, Mar. 1979.

[15] N. Kakimoto, Y. Ohsawa, and M. Hayashi, "Tansient Stability Analysis of Multimachine Power Systems with Field Flux Decays via Lyapunov's Direct Method," IEEE Trans. Power App. Syst., vol. PAS-99, no. 5, pp. 1819-1827, Sep. 1980.

[16] M. Ribbens-Pavella and F. J. Evans, "Direct Methods for Studying Dynamics of Large-scale Electric Power Systems A Survey," Automatica, vol. 21, no. 1, pp. 1-21, Jan. 1985.

[17] H.-D. Chiang, F. F. Wu, and P. P. Varaiya, "Foundations of the Potential Energy Boundary Surface Method for Power Systern Transient Stability Analysis," IEEE Trans. Circuits Syst., vol. 35, no. 6, pp. 712-728, Jun. 1988.

[18] A. A. Fouad, V. Vittal, and T. K. Oh, "Critical Energy for Direct Transient Stability Assessment of a Multimachine Power System," IEEE Trans. Power App. Syst., vol. PAS-103, no. 8, pp. 2199-2206, Aug. 1984.

[19] H.-D. Chiang, F. F. Wu, and P. P. Varaiya, "A BCU Method for Direct Analysis of Power System Transient Stability," IEEE Trans. Power Syst., vol. 9, no. 3, pp. 1194-1208, Aug. 1994.

[20] A. R. Bergen and D.J. Hill, "A Structure Preserving Model for Power System Stability Analysis," IEEE Trans. Power App. Syst., vol. PAS-100, no. 1, pp. 25-35, Jan. 1981.

[21] Y. Zou, M.-H. Yin, and H.-D. Chiang, "Theoretical Foundation of the Controlling UEP Method for Direct Transient-Stability Analysis of Network-Preserving Power System Models," IEEE Trans. Circuits Syst. - I: Fundam. Theory Appl., vol. 50, no. 10, pp. 1324-1336, Oct. 2003.

[22] L. B. Jocic, M. Ribbens-Pavella, and D. D. Siljak, "Multimachine Power Systems: Stability, Decomposition, and Aggregation," IEEE Trans. Autom. Control, vol. AC-23, no. 2, pp. 325-332, Apr. 1978.

[23] M. Araki, M. Saeki, and B. Kondo, "Application of a New Stability criterion of Composite Systems to Multimachine Power Systems," IEEE Trans. Autom. Control, vol. AC-25, no. 3, pp. 480-483, Jun. 1980.

[24] A. N. Michel, A. A. Fouad, and V. Vittal, "Power System Transient Stability Using Individual Machine Energy Functions," IEEE Trans. Circuits Syst., vol. 30, no. 5, pp. 266-276, May 1983.

[25] O. Dahl, Electric Power Circuits. Vol. II: Power System Stability. New York: McGraw-Hill, 1938.

[26] Y. Xue and M. Pavella, "Extended equal-area criterion: an analytical ultra-fast method for transient stability assessment and preventive control of power systems," International Journal of Electrical Power \& Energy Systems, vol. 11, no. 2, pp. 131-149, Apr. 1989.

[27] Y. Zhang et al., "SIME: A hybrid approach to fast transient stability assessment and contingency selection," Electrical Power \& Energy Systems, vol. 19, no. 3, pp. 195-208, Mar. 1997.

[28] D. Ernst et al., "A Unified Approach to Transient Stability Contingency Filtering, Ranking and Assessment," IEEE Trans. Power Syst., vol. 16, no. 3, pp. 435-443, Aug. 2001.

[29] D. Ruiz-Vega and M. Pavella, "A Comprehensive Approach to Transient Stability Control: Part I - Near Optimal Preventive Control," IEEE Trans. Power Syst., vol. 18, no. 4, pp. 1446-1453, Nov. 2003.

[30] M. Pavella, D. Ernst, and D. Ruizvega, Transient Stability of Power Systems: A Unified Approach to Assessment and Control. Kluwer Academic Publishers, 2000.

[31] C.-W. Liu and J. Thorp, "Application of synchronised phasor measurements to real-time transient stability prediction," Generation, Transmission and Distribuion, IEE Proceedings, vol. 142, no. 4, pp. 355-360, Jul. 1995.

[32] C.-W. Liu and J. S. Thorp, "New Methods for Computing Power System Dynamic Response for Real-Time Transient Stability Prediction," IEEE Trans. Circuits Syst. - I: Fundam. Theory Appl., vol. 47, no. 3, pp. 324-337, Mar. 2000.

[33] L. Wehenkel, T. Van Cutsem, and M. Ribbens-Pavella, "Decision Trees applied to on-line Transient Stability Assessment of Power Systems," in IEEE International Symposium on Circuits and Systems, 1988., vol. 2, Jun. 1988, pp. 1887-1890.
[34] — , "An Artificial Intelligence Framework for on-line Transient Stability Assessment of Power Systems," IEEE Trans. Power Syst., vol. 4, no. 2, pp. 789-800, May 1989.

[35] H.-Z. Guo et al., "Study on power system transient instability detection based on wide area measurement system," European Transactions on Electrical Power, vol. 20, no. 2, pp. 184-205, Mar. 2010.

[36] V. Kolluri et al., "Fast Fault Screening Approach to Assessing Transient Stability in Entergy's Power System," in Power Engineering Society General Meeting, 2007. IEEE, Jun. 2007, pp. 1-6.

[37] A. A. Fouad and V. Vittal, "The transient energy function method," International Journal of Electrical Power \& Energy Systems, vol. 10, no. 4, pp. 233-246, Oct. 1988.

[38] J. D. Meiss, Differential Dynamical Systems, A. Aceves et al., Eds. SIAM, 2007, vol. 1

[39] H.-D. Chiang, F. F. Wu, and P. P. Varaiya, "Foundations of Direct Methods for Power System Transient Stability Analysis," IEEE Trans. Circuits Syst., vol. 34, no. 2, pp. 160-173, Feb. 1987.

[40] P. P. Varaiya, F. F. Wu, and R.-L. Chen, "Direct Methods for Transient Stability Analysis of Power Systems: Recent Results," in Proceedings of the IEEE, vol. 73, no. 12, Dec. 1985, pp. 1703-1715.

[41] A. A. Fouad and S. E. Stanton, "Transient Stability of a Multi-Machine Power System Part I: Investigation of System Trajectories," IEEE Trans. Power App. Syst., vol. PAS-100, no. 7, pp. 3408-3416, Jul. 1981.

[42] P. Sauer and M. A. Pai, Power System Dynamics and Stability, E. Svendson, M. Horton, and B. M. de Leon, Eds. Prentice Hall, 1998.

[43] A. A. Fouad and S. E. Stanton, "Transient Stability of a Multi-Machine Power System Part II: Critical Transient Energy," IEEE Trans. Power App. Syst., vol. PAS-100, no. 7, pp. 3417-3424, Jul. 1981.

[44] P. Kundur, Power System Stability and Control, N. J. Balu and M. G. Lauby, Eds. McGraw-Hill Inc., 1994.

[45] J. Kleinberg and E. Tardos, Algorithm Design, M. Suarez-Rivas, Ed. Boston: Pearson Addison Wesley, 2006.

[46] F. Dorfler and F. Bullo, "Kron Reduction of Graphs with Applications to Electrical Networks," Dynamical Systems, pp. 1-28, Feb. 2011. [Online]. Available: http://arxiv.org/abs/1102.2950

[47] K. Takahishi, J. Fagan, and M. S. Chen, "Formation of a sparse bus admittance matrix and its application to short circuit study," 8th PICA Conference Proceedings, pp. 63-69, 1973.

[48] W. M. Chan and A. George, "A linear time implementation of the reverse cuthill-McKee Algorithm," BIT Numerical Mathematics, vol. 20, no. 1, pp. 8-14, Aug. 1980

[49] H. Niessner and K. Reichert, "On computing the inverse of a Sparse Matrix," International Journal for Numerical Methods in Engineering, vol. 19, no. 10, pp. 1513-1526, Oct. 1983.

\section{BIOGRAPHIES}

Tilman Weckesser (S'12) received the M.Sc. degree in sustainable energy engineering from the Technical University of Denmark (DTU), Lyngby, in 2011. He is currently a Ph.D. student at the Centre for Electric Technology (CET), DTU

His research interests are in the field of power system stability with a focus on the development of fast stability assessment methods.

Hjörtur Jóhannsson (M'10) received the M.Sc. and the PhD degree in Electrical Engineering from Technical University of Denmark, in 2007 and 2011 respectively. He is currently an assistant professor at CET, Department of Electrical Engineering, Technical University of Denmark.

His research interests concern the development of methods that provide an early warning for instability in electric power systems, and power systems dynamics, stability and control.

Stefan Sommer (S'11) received the M.Sc. degree in Mathematics in 2008 and the Ph.D. degree in Computer Science in 2012, both from the University of Copenhagen (UCPH). He is currently holding a position as postdoctoral researcher at the Technical University of Denmark (DTU), CET.

His research interests cover computational aspects of power system stability and control with focus on numerical algorithms and mathematical modeling.

Jacob Østergaard (M'95-SM'09) received the M.Sc. degree in electrical engineering from the Technical University of Denmark (DTU), Lyngby, in 1995. He was with the Research Institute of Danish Electric Utilities for 10 years. Since 2005 he has been Professor and Head of Centre for Electric Technology, DTU.

His research interests cover smart grids with focus on system integration of renewable energy and distributed energy resources, control architecture for future power system, and flexible demand. 